\title{
Macroscopic enzyme histochemistry in myocardial infarction: artefactual nature of the creatine phosphokinase reaction
}

\author{
NW DERIAS, CWM ADAMS \\ From the Department of Pathology, Guy's Hospital Medical School, St Thomas's Street, London SE1 9RT
}

SUMMARY The histochemical creatine phosphokinase (CPK) tetrazolium test has been evaluated to detect recent human myocardial infarction in gross slices of the heart at necropsy. The demonstration of the lesion with this method has been assumed to result from focal loss of CPK from the damaged myocardium. However, the present study indicates that the mechanism involved depends on localising NADPH tetrazolium reductase and not CPK. Phenazine methosulphate (PMS), when added to the incubating medium as an electron-acceptor to circumvent the tetrazolium-reductase (diaphorase) system, resulted in generalised false staining of the heart slice.

In the laboratory diagnosis of acute myocardial infarction, the serum activity of CPK often rises within three to six hours of infarction. ${ }^{1}$ Its release into the circulation has been correlated with histologically demonstrable myocardial necrosis. ${ }^{2}$ A recent histochemical study with $\mathrm{CPK}$ revealed an early macroscopic loss of enzyme activity in experimental myocardial infarction in dogs. ${ }^{3}$ However, the method has not been applied to infarcted human myocardium at the macroscopic level.

In the present work, we used the histochemical CPK method on transversely-cut slices of human heart to assess its possible use in gross identification of early human myocardial infarction at necropsy.

\section{Material and methods}

Slices of myocardium were obtained at necropsy from 47 subjects, comprising 34 cases of recent myocardial infarction and 13 road traffic accidents (Department of Forensic Medicine, Guy's Hospital Medical School). In the cases of myocardial infarction death occurred within six hours of the onset of clinical symptoms. The death-necropsy interval in all cases varied from 3-48 h.

Heart slices of approximately $1 \mathrm{~cm}$ thickness were used for the gross histochemical study of myocardial CPK activity in the normal and infarcted heart. The histochemical method employed was the tetrazolium method of Sjövall, ${ }^{4}$ originally designed for localising the enzyme in rat and human skeletal muscle, using fresh cryostat sections. The localisation of CPK activity is based on a dehydrogenation reaction resulting in the formation of blue-coloured formazan through reduction of nitroblue tetrazolium. However, the final pathway for the histochemical demonstration of CPK activity is by the way of NADPH diaphorase, according to the formulations set out by Sjövall. ${ }^{4}$

After a rinse in cold running water to remove blood, the heart slices were incubated at room temperature for $30 \mathrm{~min}$ in equal parts of the following medium: triethanolamine-acetate buffer $(0.2 \mathrm{M} \mathrm{pH} \mathrm{6.9),} \mathrm{magnesium} \mathrm{acetate} 0.02 \mathrm{M}$ and glucose $0.02 \mathrm{M}$. To $30 \mathrm{ml}$ of this solution were added: creatine phosphate $36 \mathrm{mg}$, adenosine $5^{\prime}$-diphosphate $12 \mathrm{mg}$, nicotinamide adenine dinucleotide phosphate (NADP) $6 \mathrm{mg}$, hexokinase $15 \mu \mathrm{l} 60 \mathrm{mg}$, glucose-5phosphate dehydrogenase $15 \mu 130 \mathrm{mg}$, nitroblue tetrazolium (NBT) $3 \mathrm{mg}$. Sodium cyanide was added to a concentration of $0.5 \%$ to block the respiratory chain.

To test whether deposition of the final reaction product is demonstrating the activity of CPK, heart slices were incubated in the incubating medium for CPK, but the specific substrate creatine phosphate was omitted. Heart slices were also incubated in the above incubating media with and without addition of phenazine methosulphate (PMS $0 \cdot 1 \mathrm{mg} / \mathrm{ml}$ ).

\section{Results}

Normal myocardium showed high CPK activity, as shown by homogeneous dark blue staining of the 


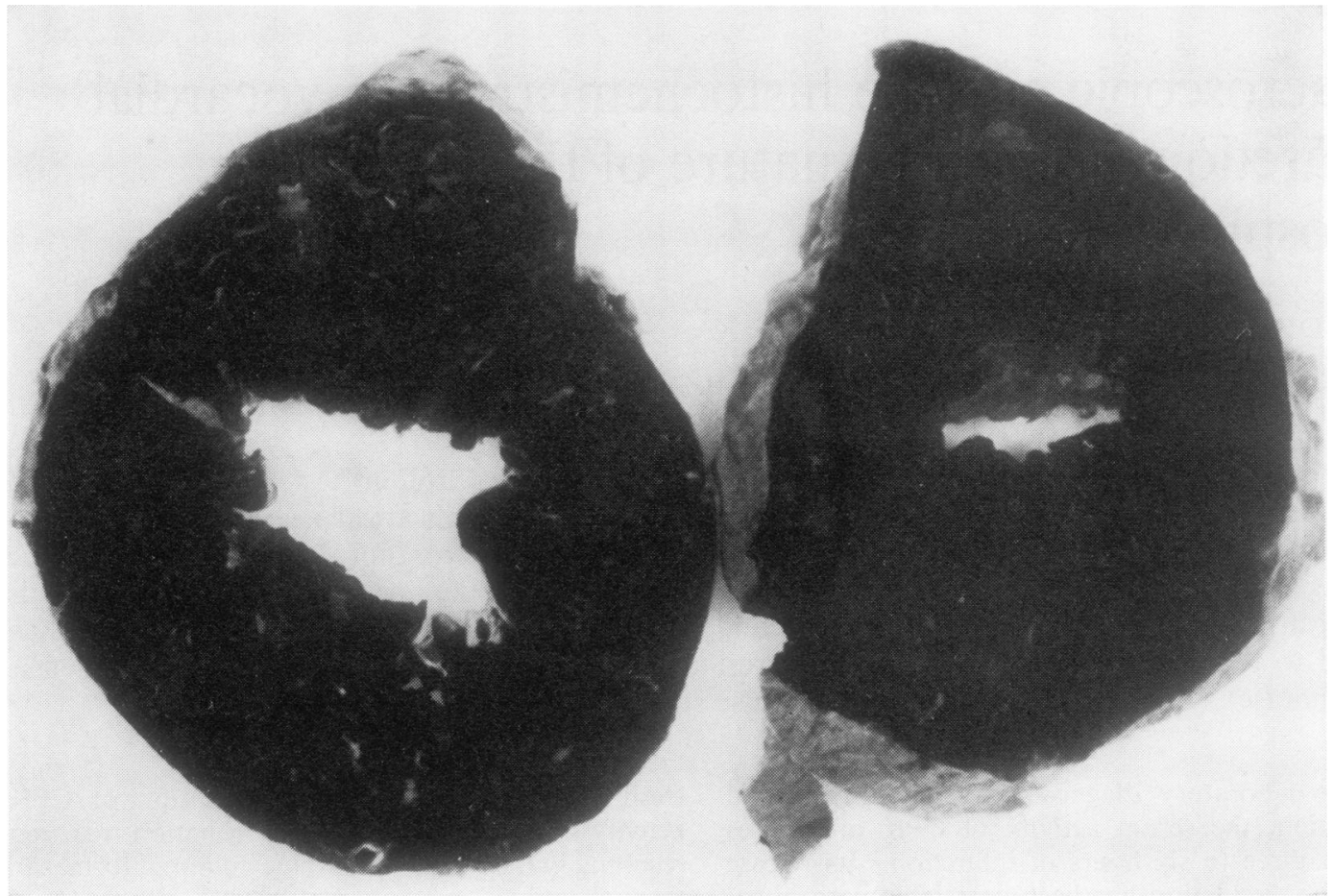

Fig. 1 Two slices from the same heart incubated in the tetrazolium incubating media for the demonstration of creatine phosphokinase activity. The substrate creatine phosphate was added to the incubating medium for the right slice, but was omitted with the left slice. The same macrostaining is observed in both heart slices.

heart muscle. However, an identical macroreaction was observed when an opposing heart slice to the one examined for CPK activity was incubated in the same incubating medium but with omission of the specific substrate creatine phosphate (Fig. 1). Identical macroscopic reactions were also observed when opposing heart slices, in which a recent myocardial infarction was suspected, were examined for CPK activity, using the tetrazolium incubating medium with and without creatine phosphate (Fig. 2). Thus, it would seem that the histochemical localisation of CPK is the same as that obtained with the endogenous-substrate nitroblue-tetrazolium method. 56

As the CPK reaction depends on endogenous NADPH diaphorase activity, elimination of this last stage was tried by adding PMS to the incubating medium, to allow the diaphorase system to be bypassed, and to bring about direct reduction of the nitroblue tetrazolium. The result, however, was unsatisfactory as generalised false staining of the heart slice was observed, as is also seen when PMS is added in the endogenous substrate-nitroblue tetrazolium method. ${ }^{6}$

\section{Discussion}

A recent histochemical study with the CPKtetrazolium method in experimental canine myocardial infarction revealed an early macroscopic loss of enzyme activity following coronary artery ligation. ${ }^{3}$ For the reasons discussed above, this method would seem also to demonstrate NADPH tetrazolium reductase. This was essentially confirmed when identical reactions were obtained when opposing heart slices, from normal and infarcted hearts, were incubated in CPK incubating medium with and without the specific substrate creatine phosphate. However, it cannot be ruled out that some other endogenous substrate may have contributed to this result (so-called "nothing dehydrogenase"). Nevertheless, this slight uncertainty does not affect the conclusion that the CPK histochemical reaction does not necessarily demonstrate creatine phosphokinase under these conditions.

The addition of PMS to obviate the diaphorase system resulted in false staining of the heart slice, as was also noted when PMS was added to the NBT incubating medium for dehydrogenases and 

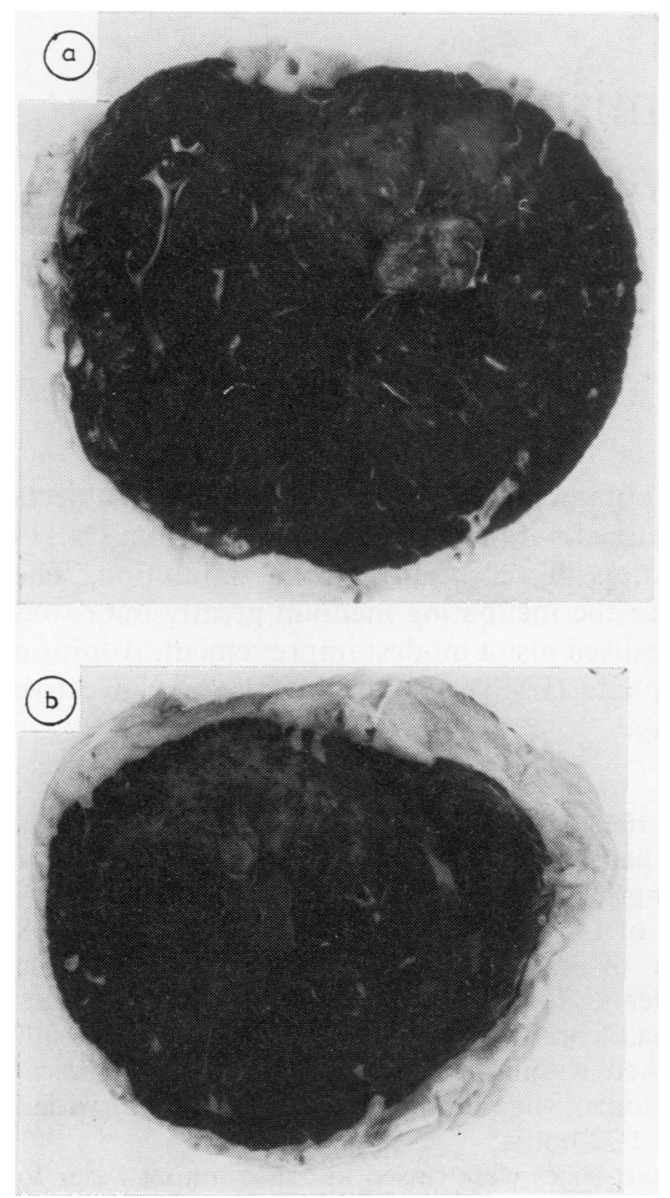

Fig. 2 Two heart slices from a 67-year-old woman with a $5 \frac{1}{2}$ hour clinical history of recent myocardial infarction. A similar macroreaction is observed in the top slice (NADPH tetrazolium reductase) as in the bottom slice (creatine phosphokinase). The recently damaged myocardium appears as focal posteroseptal diminution in the enzymatic macrostaining of the left ventricle. Occlusion of the right coronary artery by a recent thrombus. diaphorases.

In conclusion, the CPK method appears to macroscopically delineate infarction, but the mechanism involved seems to be non-specific and is not necessarily due to specific loss of CPK.

\section{References}

${ }^{1}$ Hess JW, MacDonald RP, Frederick RJ, Jones RN, Neely J, Gross D. Serum creatine phosphokinase activity in disorders of heart and skeletal muscle. Ann Intern Med 1964;61:1015-28.

${ }^{2}$ Kjekshus JK. Assessment of myocardial injury with creatine phosphokinase. Circulation 1976;53,suppl1:1-108.

${ }^{3}$ Anderson KR, Popple A, Parker DJ, Sayer R, Trickey RJ, Davies MJ. An experimental assessment of macroscopic enzyme techniques for the autopsy demonstration of myocardial infarction. J Pathol 1979;127:93-8.

${ }^{4}$ Sjövall K. A tetrazolium technique for the histochemical localization of ATP: creatine phosphotranseferase. Pattern in rat and human skeletal muscle. Histochemie 1967;10:336-40.

${ }^{5}$ Derias NW, Adams CWM. Nitroblue tetrazolium test: early gross detection of human myocardial infarcts. $\mathrm{Br} J$ Exp Pathol 1978;59:254-8.

${ }^{6}$ Derias NW, Adams CWM. Macroscopic enzyme histochemistry in myocardial infarction: use of coenzyme and phenazine methosulphate. J Clin Pathol 1982;35:'410-413

Requests for reprints to: Professor CWM Adams, Department of Pathology, Guy's Hospital Medical School, St Thomas's Street, London SE1 9RT, England. 